\title{
Yamada-Watanabe theorem for stochastic evolution equations in infinite dimensions
}

\author{
M.Röckner ${ }^{1,2}$, B.Schmuland ${ }^{3}$, X.Zhang ${ }^{4}$ \\ ${ }^{1}$ Department of Mathematics and BiBoS, Bielefeld University, Bielefeld, Germany \\ 2 Departments of Mathematics and Statistics, Purdue University, West Lafayette, USA \\ 3 Department of Mathematical and Statistical Sciences, University of Alberta, Edmonton, Canada \\ ${ }^{4}$ Department of Statistics, School of Mathematics and Statistics, University of New South Wales, Sydney, \\ Australia
}

Received January 31, 2008

The purpose of this note is to give a complete and detailed proof of the fundamental Yamada-Watanabe Theorem on infinite dimensional spaces, more precisely in the framework of the variational approach to stochastic partial differential equations.

Key words: integration by parts, stochastic dynamics

PACS: $02.03 . \mathrm{Sa}, 02.50 . \mathrm{Ey}$

\section{Framework and definitions}

Let $H$ be a separable Hilbert space, with inner product $\langle\cdot, \cdot\rangle_{\mathrm{H}}$ and norm $\|\cdot\|_{\mathrm{H}}$. Let $V, E$ be separable Banach spaces with norms $\|\cdot\|_{V}$ and $\|\cdot\|_{E}$, such that

$$
V \subset H \subset E
$$

continuously and densely. For a topological space $X$ let $\mathcal{B}(X)$ denote its Borel $\sigma$-algebra. By Kuratowski's theorem we have that $V \in \mathcal{B}(H), H \in \mathcal{B}(E)$ and $\mathcal{B}(V)=\mathcal{B}(H) \cap V, \mathcal{B}(H)=\mathcal{B}(E) \cap H$.

Setting $\|x\|_{V}:=\infty$ if $x \in H \backslash V$, we extend $\|\cdot\|_{V}$ to a function on $H$. We recall that this extension is $\mathcal{B}(H)$-measurable and lower semicontinuous (cf. e.g. [4, Exercise 4.2.3]). Hence the following path space is well-defined:

$$
\mathbb{B}:=\left\{w \in C\left(\mathbb{R}_{+} ; H\right) \mid \int_{0}^{T}\|w(t)\|_{V} \mathrm{~d} t<\infty \text { for all } T \in[0, \infty)\right\},
$$

equipped with the metric

$$
\rho\left(w_{1}, w_{2}\right):=\sum_{k=1}^{\infty} 2^{-k}\left[\left(\int_{0}^{k}\left\|w_{1}(t)-w_{2}(t)\right\|_{V} \mathrm{~d} t+\sup _{t \in[0, k]}\left\|w_{1}(t)-w_{2}(t)\right\|_{\mathrm{H}}\right) \wedge 1\right] .
$$

Obviously, $(\mathbb{B}, \rho)$ is a complete separable metric space. Let $\mathcal{B}_{t}(\mathbb{B})$ denote the $\sigma$-algebra generated by all maps $\pi_{s}: \mathbb{B} \rightarrow H, s \in[0, t]$, where $\pi_{s}(w):=w(s), w \in \mathbb{B}$. For $t \geqslant 0$ and $w \in \mathbb{B}$ define the stopped path $w^{t}$ by

$$
w^{t}(s)=w(s \wedge t), \quad s \geqslant 0 .
$$

Below we shall use the following elementary, but useful measure theoretic facts.

Lemma 1.1. The map $w \mapsto w^{t}$ is $\mathcal{B}_{t}(\mathbb{B}) / \mathcal{B}(\mathbb{B})$ measurable.

Proof. It suffices to show that $\pi_{q}\left(w^{t}\right)$ is $\mathcal{B}_{t}(\mathbb{B}) / \mathcal{B}(H)$ measurable for $q \geqslant 0$. But $\pi_{q}\left(w^{t}\right)=\pi_{q}(w)$ if $q \leqslant t$, and $\pi_{q}\left(w^{t}\right)=\pi_{t}(w)$ otherwise. In either case, we get a $\mathcal{B}_{t}(\mathbb{B}) / \mathcal{B}(H)$ measurable map. 
Lemma 1.2. For every set $A \in \mathcal{B}_{t}(\mathbb{B})$ we have $1_{A}(w)=1_{A}\left(w^{t}\right)$.

Proof. By definition, $1_{A}(w)=1_{A}\left(w^{t}\right)$ for sets of the form

$$
A=\left\{w: \pi_{s_{1}}(w) \in B_{1}, \ldots, \pi_{s_{n}}(w) \in B_{n}\right\}
$$

for $n \geqslant 1, s_{i} \leqslant t$, and $B_{i} \in \mathcal{B}(H)$. Since these sets generate $\mathcal{B}_{t}(\mathbb{B})$, the monotone class theorem finishes the proof.

Corollary 1.3. Let $(E, \mathcal{E})$ be a measurable space and $f: \mathbb{B} \rightarrow E$ a $\mathcal{B}(\mathbb{B}) / \mathcal{E}$ measurable map. Then $f$ is $\mathcal{B}_{t}(\mathbb{B}) / \mathcal{E}$ measurable if and only if $f(w)=f\left(w^{t}\right)$ for all $w \in \mathbb{B}$.

Let $\left(U,\langle,\rangle_{U}\right)$ be another separable Hilbert space and let $L_{2}(U, H)$ denote the space of all Hilbert-Schmidt operators from $U$ to $H$ equipped with the usual Hilbert-Schmidt norm \|\|$_{L_{2}}$.

Let $b: \mathbb{R}_{+} \times \mathbb{B} \rightarrow E$ and $\sigma: \mathbb{R}_{+} \times \mathbb{B} \rightarrow L_{2}(U, H)$ be $\mathcal{B}\left(\mathbb{R}_{+}\right) \otimes \mathcal{B}(\mathbb{B}) / \mathcal{B}(E)$ and $\mathcal{B}\left(\mathbb{R}_{+}\right) \otimes$ $\mathcal{B}(\mathbb{B}) / \mathcal{B}\left(L_{2}(U, H)\right)$-measurable respectively such that for each $t \in \mathbb{R}_{+}$

$$
b(t, \cdot) \text { is } \mathcal{B}_{t}(\mathbb{B}) / \mathcal{B}(E) \text {-measurable }
$$

and

$$
\sigma(t, \cdot) \text { is } \mathcal{B}_{t}(\mathbb{B}) / \mathcal{B}\left(L_{2}(U, H)\right) \text {-measurable. }
$$

As usual we call $\left(\Omega, \mathcal{F}, P,\left(\mathcal{F}_{t}\right)\right)$ a stochastic basis if $(\Omega, \mathcal{F}, P)$ is a complete probability space and $\left(\mathcal{F}_{t}\right)$ is a right continuous filtration on $\Omega$ augmented by the $P$-zero sets. Let $\beta_{k}, k \in \mathbb{N}$, be independent $\left(\mathcal{F}_{t}\right)$-Brownian motions on a stochastic basis $\left(\Omega, \mathcal{F}, P,\left(\mathcal{F}_{t}\right)\right)$ and define the sequence

$$
W(t):=\left(\beta_{k}(t)\right)_{k \in \mathbb{N}}, \quad t \in[0, \infty) .
$$

Below we refer to such a process $W$ on $\mathbb{R}^{\infty}$ as standard $\mathbb{R}^{\infty}$-Wiener process. We fix an orthonormal basis $\left\{e_{k}, k \in \mathbb{N}\right\}$ of $U$ and consider $W$ as a cylindrical Wiener process on $U$, that is, we informally have

$$
W(t)=\sum_{k=1}^{\infty} \beta_{k}(t) e_{k}, \quad t \in[0, \infty) .
$$

We consider the following stochastic evolution equation:

$$
\mathrm{d} X(t)=b(t, X) \mathrm{d} t+\sigma(t, X) \mathrm{d} W(t), \quad t \in[0, \infty) .
$$

Definition 1.4. A pair $(X, W)$, where $X=(X(t))_{t \in[0, \infty)}$ is an $\left(\mathcal{F}_{t}\right)$-adapted process with paths in $\mathbb{B}$ and $W$ is a standard $\mathbb{R}^{\infty}$-Wiener process on a stochastic basis $\left(\Omega, \mathcal{F}, P,\left(\mathcal{F}_{t}\right)\right)$ is called a weak solution of (1.1) if

(i) For any $T \in[0, \infty)$

$$
\int_{0}^{T}\|b(s, X)\|_{E} \mathrm{~d} s+\int_{0}^{T}\|\sigma(s, X)\|_{L_{2}(U, H)}^{2} \mathrm{~d} s<\infty \quad P \text {-a.e. }
$$

(ii) As a stochastic equation on $E$ we have

$$
X(t)=X(0)+\int_{0}^{t} b(s, X) \mathrm{d} s+\int_{0}^{t} \sigma(s, X) \mathrm{d} W(s), \quad t \in[0, \infty) \quad P \text {-a.e. }
$$

Remark 1.5. (i) By the measurability assumptions on $b$ and $\sigma$, it follows that if $X$ is as in Definition 1.4 then both processes $b(\cdot, X)$ and $\sigma(\cdot, X)$ are $\left(\mathcal{F}_{t}\right)$-adapted. 
(ii) We recall that by definition of the $H$-valued stochastic integral in (ii) we have

$$
\int_{0}^{t} \sigma(s, X) \mathrm{d} W(s):=\int_{0}^{t} \sigma(s, X) \circ J^{-1} \mathrm{~d} \bar{W}(s), \quad t \in[0, \infty),
$$

where $J$ is any one-to-one Hilbert-Schmidt operator from $U$ into another Hilbert space $\left(\bar{U}\langle,\rangle_{\bar{U}}\right)$ and

$$
\bar{W}(t):=\sum_{k=1}^{\infty} \beta_{k}(t) J e_{k}, \quad t \in[0, \infty) .
$$

This definition of the stochastic integral is independent of the choice of $J$ and $\left(\bar{U},\langle,\rangle_{\bar{U}}\right)$. We refer e.g. to [4, Section 2.5] for details, and only mention here that for $s \in[0, \infty), w \in \mathbb{B}$

$$
\begin{gathered}
\sigma(s, w) \circ J^{-1} \in L_{2}\left(Q^{1 / 2}(\bar{U}), H\right) \\
\text { with }\left\|\sigma(s, w) \circ J^{-1}\right\|_{L_{2}\left(Q^{1 / 2}(\bar{U}), H\right)}=\|\sigma(s, w)\|_{L_{2}(U, H)},
\end{gathered}
$$

where $Q:=J J^{*}$, and that $\bar{W}$ is a $Q$-Wiener process on $\bar{U}$.

Below we shall fix one such $J$ and $\left(\bar{U},\langle,\rangle_{\bar{U}}\right)$ as in Remark 1.5(ii) and set

$$
\bar{\sigma}(s, w):=\sigma(s, w) \circ J^{-1}, \quad s \in[0, \infty), w \in \mathbb{B},
$$

and for any standard $\mathbb{R}^{\infty}$-Wiener process $W$ we define $\bar{W}$ as in (1.2) for the fixed $J$. Furthermore, we define

$$
\mathbb{W}_{0}:=\left\{w \in C\left(\mathbb{R}_{+}, \bar{U}\right) \mid w(0)=0\right\}
$$

equipped with the supremum norm and Borel $\sigma$-algebra $\mathcal{B}\left(\mathbb{W}_{0}\right)$. For $t \in \mathbb{R}_{+}$let $\mathcal{B}_{t}\left(\mathbb{W}_{0}\right)$ be the $\sigma$-algebra generated by $\pi_{s}: \mathbb{W}_{0} \rightarrow \bar{U}, 0 \leqslant s \leqslant t, \pi_{s}(w):=w(s)$.

Definition 1.6. We say that weak uniqueness holds for $(1.1)$ if whenever $(X, W)$ and $\left(X^{\prime}, W^{\prime}\right)$ are two weak solutions with stochastic bases $\left(\Omega, \mathcal{F}, P,\left(\mathcal{F}_{t}\right)\right)$ and $\left(\Omega^{\prime}, \mathcal{F}^{\prime}, P^{\prime},\left(\mathcal{F}_{t}^{\prime}\right)\right)$ such that

$$
P \circ X(0)^{-1}=P^{\prime} \circ X^{\prime}(0)^{-1}
$$

(as measures on $(H, \mathcal{B}(H)))$, then

$$
P \circ X^{-1}=P^{\prime} \circ\left(X^{\prime}\right)^{-1}
$$

(as measures on $(\mathbb{B}, \mathcal{B}(\mathbb{B}))$ ).

Definition 1.7. We say that pathwise uniqueness holds for $(1.1)$, if whenever $(X, W),\left(X^{\prime}, W^{\prime}\right)$ are two weak solutions on the same stochastic basis $\left(\Omega, \mathcal{F}, P,\left(\mathcal{F}_{t}\right)\right)$ and with the same standard$\mathbb{R}^{\infty}$-Wiener process $W$ on $(\Omega, \mathcal{F}, P)$ such that $X(0)=X^{\prime}(0) P$-a.e., then $P$-a.e.

$$
X(t)=X^{\prime}(t), t \in[0, \infty) .
$$

To define strong solutions we need to introduce the following class $\hat{\mathcal{E}}$ of maps:

Let $\hat{\mathcal{E}}$ denote the set of all maps $F: H \times \mathbb{W}_{0} \rightarrow \mathbb{B}$ such that for every probability measure $\mu$ on $(H, \mathcal{B}(H))$ there exists a $\overline{\mathcal{B}(H) \otimes \mathcal{B}\left(\mathbb{W}_{0}\right)}{ }^{\mu \otimes P^{Q}} / \mathcal{B}(\mathbb{B})$-measurable map $F_{\mu}: H \times \mathbb{W}_{0} \rightarrow \mathbb{B}$ such that for $\mu$-a.e. $x \in H$

$$
F(x, w)=F_{\mu}(x, w) \text { for } P^{Q} \text {-a.e. } w \in \mathbb{W}_{0} .
$$

Here $\overline{\mathcal{B}(H) \otimes \mathcal{B}\left(\mathbb{W}_{0}\right)}{ }^{\mu \otimes P^{Q}}$ denotes the completion of $\mathcal{B}(H) \otimes \mathcal{B}\left(\mathbb{W}_{0}\right)$ with respect to $\mu \otimes P^{Q}$, and $P^{Q}$ denotes the distribution of the $Q$-Wiener process on $\bar{U}$ on $\left(\mathbb{W}_{0}, \mathcal{B}\left(\mathbb{W}_{0}\right)\right)$. Of course, $F_{\mu}$ is uniquely determined $\mu \otimes P^{Q}$-a.e. 
Definition 1.8. A weak solution $(X, W)$ to $(1.1)$ on $\left(\Omega, \mathcal{F}, P,\left(\mathcal{F}_{t}\right)\right)$ is called a strong solution if there exists $F \in \hat{\mathcal{E}}$ such that for $x \in H, w \mapsto F(x, w)$ is $\overline{\mathcal{B}_{t}\left(\mathbb{W}_{0}\right)} P^{Q} / \mathcal{B}_{t}(\mathbb{B})$-measurable for every $t \in[0, \infty)$ and

$$
X=F_{P \circ X(0)^{-1}}(X(0), \bar{W}) \quad P \text {-a.e. }
$$

where ${\overline{\mathcal{B}_{t}\left(\mathbb{W}_{0}\right)}}^{Q}$ denotes the completion with respect to $P^{Q}$ in $\mathcal{B}\left(\mathbb{W}_{0}\right)$.

Definition 1.9. Equation (1.1) is said to have a unique strong solution, if there exists $F \in \hat{\mathcal{E}}$ satisfying the adaptiveness condition in Definition 1.8 and such that:

1. For every standard $\mathbb{R}^{\infty}$-Wiener process on a stochastic basis $\left(\Omega, \mathcal{F}, P,\left(\mathcal{F}_{t}\right)\right)$ and any $\mathcal{F}_{0} / \mathcal{B}(H)$ measurable $\xi: \Omega \rightarrow H$ the continuous process

$$
X:=F_{P \circ \xi^{-1}}(\xi, \bar{W})
$$

is $\left(\mathcal{F}_{t}\right)$-adapted and satisfies (i), (ii) in Definition 1.4, i.e. $(F(\xi, \bar{W}), W)$ is a weak solution to $(1.1)$, and $X(0)=\xi P$-a.e.

2. For any weak solution $(X, W)$ to $(1.1)$ we have

$$
X=F_{P \circ X(0)^{-1}}(X(0), \bar{W}) P \text {-a.e. }
$$

Remark 1.10. Since $X(0)$ of a weak solution is P-independent of $\bar{W}$, thus

$$
P \circ(X(0), \bar{W})^{-1}=\mu \otimes P^{Q},
$$

we have that the existence of a unique strong solution for (1.1) implies that also weak uniqueness holds.

\section{The main result and its proof}

Let us now formulate the main result (see e.g. [2] for the finite dimensional case).

Theorem 2.1. Let $\sigma$ and $b$ be as above. Then equation (1.1) has a unique strong solution if and only if both of the following properties hold:

(i) For every probability measure $\mu$ on $(H, \mathcal{B}(H))$ there exists a weak solution $(X, W)$ of $(1.1)$ such that $\mu$ is the distribution of $X(0)$.

(ii) Pathwise uniqueness holds for (1.1).

Proof. Suppose (1.1) has a unique strong solution. Then (ii) obviously holds. To show (i) one only has to take the probability space $\left(\mathbb{W}_{0}, \mathcal{B}\left(\mathbb{W}_{0}\right), P^{Q}\right)$ and consider $\left(H \times \mathbb{W}_{0}, \overline{\mathcal{B}(H) \otimes \mathcal{B}\left(\mathbb{W}_{0}\right)} \mu \otimes P^{Q}, \mu \otimes\right.$ $\left.P^{Q}\right)$ with filtration

$$
\bigcap_{\varepsilon>0} \sigma\left(\mathcal{B}(H) \otimes \mathcal{B}_{t+\varepsilon}\left(\mathbb{W}_{0}\right), \mathcal{N}\right), \quad t \geqslant 0
$$

where $\mathcal{N}$ denotes all $\mu \otimes P^{Q}$-zero sets in $\overline{\mathcal{B}(H) \otimes \mathcal{B}\left(\mathbb{W}_{0}\right)}{ }^{\mu \otimes P^{Q}}$. Let $\xi: H \times \mathbb{W}_{0} \rightarrow H$ and $W: H \times \mathbb{W}_{0} \rightarrow \mathbb{W}_{0}$ be the canonical projections. Then $X:=F_{P \circ \xi^{-1}}(\xi, W)$ is the desired weak solution in (i).

Now let us suppose that (i) and (ii) hold. The proof that then there exists a unique strong solution for (1.1) is quite technical. We structure it through a series of lemmas. 
Lemma 2.2. Let $(\Omega, \mathcal{F})$ be a measurable space such that $\{\omega\} \in \mathcal{F}$ for all $\omega \in \Omega$ and such that

$$
D:=\{(\omega, \omega) \mid \omega \in \Omega\} \in \mathcal{F} \otimes \mathcal{F}
$$

(which is e.g. the case if $\Omega$ is a Polish space and $\mathcal{F}$ its Borel $\sigma$-algebra). Let $P_{1}, P_{2}$ be probability measures on $(\Omega, \mathcal{F})$ such that $P_{1} \otimes P_{2}(D)=1$. Then $P_{1}=P_{2}=\delta_{\omega_{0}}$ for some $\omega_{0} \in \Omega$.

Proof. Let $f: \Omega \rightarrow[0, \infty)$ be $\mathcal{F}$-measurable. Then

$$
\begin{aligned}
\int f\left(\omega_{1}\right) P_{1}\left(\mathrm{~d} \omega_{1}\right) & =\iint f\left(\omega_{1}\right) P_{1}\left(\mathrm{~d} \omega_{1}\right) P_{2}\left(\mathrm{~d} \omega_{2}\right)=\iint 1_{D}\left(\omega_{1}, \omega_{2}\right) f\left(\omega_{1}\right) P_{1}\left(\mathrm{~d} \omega_{1}\right) P_{2}\left(\mathrm{~d} \omega_{2}\right) \\
& =\iint 1_{D}\left(\omega_{1}, \omega_{2}\right) f\left(\omega_{2}\right) P_{1}\left(\mathrm{~d} \omega_{1}\right) P_{2}\left(\mathrm{~d} \omega_{2}\right)=\int f\left(\omega_{2}\right) P_{2}\left(\mathrm{~d} \omega_{2}\right)
\end{aligned}
$$

so $P_{1}=P_{2}$. Furthermore,

$$
1=\iint 1_{D}\left(\omega_{1}, \omega_{2}\right) P_{1}\left(\mathrm{~d} \omega_{1}\right) P_{2}\left(\mathrm{~d} \omega_{2}\right)=\int P_{1}\left(\left\{\omega_{2}\right\}\right) P_{2}\left(\mathrm{~d} \omega_{2}\right),
$$

hence $1=P_{1}\left(\left\{\omega_{2}\right\}\right)$ for $P_{2}$-a.e. $\omega_{2} \in \Omega$. Therefore, $P_{1}=\delta_{\omega_{0}}$ for some $\omega_{0} \in \Omega$.

Fix a probability measure $\mu$ on $(H, \mathcal{B}(H))$ and let $(X, W)$ with stochastic basis $\left(\Omega, \mathcal{F}, P,\left(\mathcal{F}_{t}\right)\right)$ be a weak solution to $(1.1)$ with initial distribution $\mu$. Define a probability measure $P_{\mu}$ on $(H \times$ $\left.\mathbb{B} \times \mathbb{W}_{0}, \mathcal{B}(H) \otimes \mathcal{B}(\mathbb{B}) \otimes \mathcal{B}\left(\mathbb{W}_{0}\right)\right)$, by

$$
P_{\mu}:=P \circ(X(0), X, \bar{W})^{-1} .
$$

Lemma 2.3. There exists a family $K_{\mu}\left((x, w), \mathrm{d} w_{1}\right), x \in H, w \in \mathbb{W}_{0}$, of probability measures on $(\mathbb{B}, \mathcal{B}(\mathbb{B}))$ having the following properties:

(i) For every $A \in \mathcal{B}(\mathbb{B})$ the map

$$
H \times \mathbb{W}_{0} \ni(x, w) \mapsto K_{\mu}((x, w), A)
$$

is $\mathcal{B}(H) \otimes \mathcal{B}\left(\mathbb{W}_{0}\right)$-measurable.

(ii) For every $\mathcal{B}(H) \otimes \mathcal{B}(\mathbb{B}) \otimes \mathcal{B}\left(\mathbb{W}_{0}\right)$-measurable map $f: H \times \mathbb{B} \times \mathbb{W}_{0} \rightarrow[0, \infty)$ we have

$$
\int_{H \times \mathbb{B} \times \mathbb{W}_{0}} f\left(x, w_{1}, w\right) P_{\mu}\left(\mathrm{d} x, \mathrm{~d} w_{1}, \mathrm{~d} w\right)=\int_{H} \int_{\mathbb{W}_{0}} \int_{\mathbb{B}} f\left(x, w_{1}, w\right) K_{\mu}\left((x, w), \mathrm{d} w_{1}\right) P^{Q}(\mathrm{~d} w) \mu(\mathrm{d} x) .
$$

(iii) If $t \in[0, \infty)$ and $f: \mathbb{B} \rightarrow[0, \infty)$ is $\mathcal{B}_{t}(\mathbb{B})$-measurable, then

$$
H \times \mathbb{W}_{0} \ni(x, w) \mapsto \int_{\mathbb{B}} f\left(w_{1}\right) K_{\mu}\left((x, w), \mathrm{d} w_{1}\right)
$$

is $\overline{\mathcal{B}(H) \otimes \mathcal{B}_{t}\left(\mathbb{W}_{0}\right)}{ }^{\mu \otimes P^{Q}}$-measurable, where $\overline{\mathcal{B}(H) \otimes \mathcal{B}_{t}\left(\mathbb{W}_{0}\right)}{ }^{\mu \otimes P^{Q}} \quad$ denotes the completion with respect to $\mu \otimes P^{Q}$ in $\mathcal{B}(H) \otimes \mathcal{B}\left(\mathbb{W}_{0}\right)$.

Proof. Let $\Pi: H \times \mathbb{B} \times \mathbb{W}_{0} \rightarrow H \times \mathbb{W}_{0}$ be the canonical projection. Since $X(0)$ is $\mathcal{F}_{0}$-measurable, hence $P$-independent of $\bar{W}$, it follows that

$$
P_{\mu} \circ \Pi^{-1}=P \circ(X(0), \bar{W})^{-1}=\mu \otimes P^{Q} .
$$

Hence by the existence result on regular conditional distributions (cf. e.g. [2, Corollary to Theorem 3.3 on p. 15]), the existence of the family $K_{\mu}\left((x, w), \mathrm{d} w_{1}\right), x \in H, w \in \mathbb{W}_{0}$, satisfying (i) and (ii) follows. 
To prove (iii) it suffices to show that for $t \in[0, \infty)$ and for all $A_{0} \in \mathcal{B}(H), A_{1} \in \mathcal{B}_{t}(\mathbb{B})$, $A \in \mathcal{B}_{t}\left(\mathbb{W}_{0}\right)$ and

$$
\begin{gathered}
A^{\prime}:=\left\{\pi_{r_{1}}-\pi_{t} \in B_{1}, \pi_{r_{2}}-\pi_{r_{1}} \in B_{2}, \ldots, \pi_{r_{k}}-\pi_{r_{k-1}} \in B_{k}\right\}\left(\subset \mathbb{W}_{0}\right), \\
t \leqslant r_{1}<\ldots<r_{k}, B_{1}, \ldots, B_{k} \in \mathcal{B}(\bar{U}), \\
\int_{A_{0}} \int_{\mathbb{W}_{0}} 1_{A \cap A^{\prime}}(w) K_{\mu}\left((x, w), A_{1}\right) P^{Q}(\mathrm{~d} w) \mu(\mathrm{d} x) \\
=\int_{A_{0}} \int_{\mathbb{W}_{0}} 1_{A \cap A^{\prime}}(w) \mathbb{E}_{\mu \otimes P^{Q}}\left(K_{\mu}\left(\cdot, A_{1}\right) \mid \mathcal{B}(H) \otimes \mathcal{B}_{t}\left(\mathbb{W}_{0}\right)\right) P^{Q}(\mathrm{~d} w) \mu(\mathrm{d} x),
\end{gathered}
$$

since the system of all $A \cap A^{\prime}, A \in \mathcal{B}_{t}\left(\mathbb{W}_{0}\right), A^{\prime}$ as above generates $\mathcal{B}\left(\mathbb{W}_{0}\right)$. But by part (ii) above, the left-hand side of $(2.1)$ is equal to

$$
\int_{H \times \mathbb{B} \times \mathbb{W}_{0}} 1_{A_{0}}(x) 1_{A \cap A^{\prime}}(w) 1_{A_{1}}\left(w_{1}\right) P_{\mu}\left(\mathrm{d} x, \mathrm{~d} w_{1}, \mathrm{~d} w\right)=\int_{\Omega} 1_{A_{0}}(X(0)) 1_{A_{1}}(X) 1_{A}(\bar{W}) 1_{A^{\prime}}(\bar{W}) \mathrm{d} P .
$$

But $1_{A^{\prime}}(\bar{W})$ is $P$-independent of $\mathcal{F}_{t}$, since $W$ is a standard $\mathbb{R}^{\infty}$-Wiener process on $\left(\Omega, \mathcal{F}, P, \mathcal{F}_{t}\right)$, so the right-hand side of $(2.2)$ is equal to

$$
\begin{aligned}
& \int_{\Omega} 1_{A^{\prime}}(\bar{W}) \mathrm{d} P \cdot \int_{\Omega} 1_{A_{0}}(X(0)) 1_{A_{1}}(X) 1_{A}(\bar{W}) 1_{A^{\prime}}(\bar{W}) \mathrm{d} P \\
& \quad=P^{Q}\left(A^{\prime}\right) \int_{H \times \mathbb{B} \times \mathbb{W}_{0}} 1_{A_{0}}(x) 1_{A}(w) 1_{A_{1}}\left(w_{1}\right) P_{\mu}\left(\mathrm{d} x, \mathrm{~d} w_{1}, \mathrm{~d} w\right) \\
& =P^{Q}\left(A^{\prime}\right) \int_{A_{0}} \int_{A} K_{\mu}\left((x, w), A_{1}\right) P^{Q}(\mathrm{~d} w) \mu(\mathrm{d} x) \\
& =P^{Q}\left(A^{\prime}\right) \int_{A_{0}} \int_{A} \mathbb{E}_{\mu \otimes P^{Q}}\left(K_{\mu}\left(\cdot, A_{1}\right) \mid \mathcal{B}(H) \otimes \mathcal{B}_{t}\left(\mathbb{W}_{0}\right)\right)((x, w)) P^{Q}(\mathrm{~d} w) \mu(\mathrm{d} x) \\
& =\int_{A_{0}} \int_{\mathbb{W}_{0}} 1_{A \cap A^{\prime}}(w) \mathbb{E}_{\mu \otimes P^{Q}}\left(K_{\mu}\left(\cdot, A_{1}\right) \mid \mathcal{B}(H) \otimes \mathcal{B}_{t}\left(\mathbb{W}_{0}\right)\right)((x, w)) P^{Q}(\mathrm{~d} w) \mu(\mathrm{d} x),
\end{aligned}
$$

since $A^{\prime}$ is $P^{Q}$-independent of $\mathcal{B}_{t}\left(\mathbb{W}_{0}\right)$.

For $x \in H$ define a measure $Q_{x}$ on

$$
\left(H \times \mathbb{B} \times \mathbb{B} \times \mathbb{W}_{0}, \mathcal{B}(H) \otimes \mathcal{B}(\mathbb{B}) \otimes \mathcal{B}(\mathbb{B}) \otimes \mathcal{B}\left(\mathbb{W}_{0}\right)\right)
$$

by

$$
Q_{x}(A):=\int_{H} \int_{\mathbb{B}} \int_{\mathbb{B}} \int_{\mathbb{W}_{0}} 1_{A}\left(z, w_{1}, w_{2}, w\right) K_{\mu}\left((z, w), \mathrm{d} w_{1}\right) K_{\mu}\left((z, w), \mathrm{d} w_{2}\right) P^{Q}(\mathrm{~d} w) \delta_{x}(\mathrm{~d} z) .
$$

Define the stochastic basis

$$
\begin{aligned}
\tilde{\Omega} & :=H \times \mathbb{B} \times \mathbb{B} \times \mathbb{W}_{0}, \\
\tilde{\mathcal{F}}^{x} & :=\overline{\mathcal{B}(H) \otimes \mathcal{B}(\mathbb{B}) \otimes \mathcal{B}(\mathbb{B}) \otimes \mathcal{B}\left(\mathbb{W}_{0}\right)} Q^{Q_{x}}, \\
\tilde{\mathcal{F}}_{t}^{x} & :=\bigcap_{\varepsilon>0} \sigma\left(\mathcal{B}(H) \otimes \mathcal{B}_{t+\varepsilon}(\mathbb{B}) \otimes \mathcal{B}_{t+\varepsilon}\left(\mathbb{W}_{0}\right) \otimes \mathcal{B}_{t+\varepsilon}\left(\mathbb{W}_{0}\right), \mathcal{N}_{x}\right),
\end{aligned}
$$

where

$$
\mathcal{N}_{x}:=\left\{N \in \tilde{\mathcal{F}}^{x} \mid Q_{x}(N)=0\right\}
$$


and define maps

$$
\begin{aligned}
& \Pi_{0}: \tilde{\Omega} \rightarrow H,\left(x, w_{1}, w_{2}, w\right) \mapsto x, \\
& \Pi_{i}: \tilde{\Omega} \rightarrow \mathbb{B},\left(x, w_{1}, w_{2}, w\right) \mapsto w_{i} \in \mathbb{B}, \quad i=1,2, \\
& \Pi_{3}: \tilde{\Omega} \rightarrow \mathbb{W}_{0},\left(x, w_{1}, w_{2}, w\right) \mapsto w \in \mathbb{W}_{0} .
\end{aligned}
$$

Then, obviously,

$$
Q_{x} \circ \Pi_{0}^{-1}=\delta_{x}
$$

and

$$
Q_{x} \circ \Pi_{3}^{-1}=P^{Q}\left(=P \circ \bar{W}^{-1}\right) .
$$

Lemma 2.4. There exists $N_{0} \in \mathcal{B}(H)$ with $\mu\left(N_{0}\right)=0$ such that for all $x \in N_{0}^{c}$ we have that $\Pi_{3}$ is an $\left(\tilde{\mathcal{F}}_{t}^{x}\right)$-Wiener process on $\left(\tilde{\Omega}, \tilde{\mathcal{F}}^{x}, Q_{x}\right)$ taking values in $\bar{U}$.

Proof. By definition $\Pi_{3}$ is $\left(\tilde{\mathcal{F}}_{t}^{x}\right)$-adapted for every $x \in H$. Furthermore, for $0 \leqslant s<t, y \in H$, and $A_{0} \in \mathcal{B}(H), A_{i} \in \mathcal{B}_{s}(\mathbb{B}), i=1,2, A_{3} \in \mathcal{B}_{s}\left(\mathbb{W}_{0}\right)$,

$$
\begin{aligned}
\int_{H} & \mathbb{E}_{Q_{x}}\left(\exp \left(i\left\langle y, \Pi_{3}(t)-\Pi_{3}(s)\right\rangle\right) 1_{A_{0} \times A_{1} \times A_{2} \times A_{3}}\right) \mu(\mathrm{d} x) \\
= & \int_{H} \int_{\mathbb{W}_{0}} \exp (i\langle y, w(t)-w(s)\rangle) 1_{A_{0}}(x) 1_{A_{3}}(w) K_{\mu}\left((x, w), A_{1}\right) K_{\mu}\left((x, w), A_{2}\right) P^{Q}(\mathrm{~d} w) \mu(\mathrm{d} x) \\
= & \int_{\mathbb{W}_{0}} \exp (i\langle y, w(t)-w(s)\rangle) P^{Q}(\mathrm{~d} w) \int_{H} Q_{x}\left(A_{0} \times A_{1} \times A_{2} \times A_{3}\right) \mu(\mathrm{d} x),
\end{aligned}
$$

where we used Lemma 2.3(iii) in the last step. Now the assertion follows by (2.4), a monotone class argument and the same reasoning as in the proof of [4, Proposition 2.1.13].

Over the next few lemmas we carefully develop a pathwise definition of the stochastic integral. Some care is required as we need to consider the integral simultaneously with respect to several different stochastic bases. The definition of the stochastic integral uses the notions of predictability and stopping times, both of which depend on the underlying filtration. We start with an approximation to the integrand that is predictable with respect to the raw filtration.

Lemma 2.5. Let $Z$ be a separable Hilbert space valued, adapted, measurable process on a stochastic basis $\left(\Omega, \mathcal{F},\left(\mathcal{F}_{t}\right), P\right)$. If $E \int_{0}^{T}\|Z(s, \omega)\|^{2} \mathrm{~d} s<\infty$, then there exist elementary predictable processes $p_{n}$ so that

$$
E \int_{0}^{T}\left\|Z(s, \omega)-p_{n}(s, \omega)\right\|^{2} \mathrm{~d} s \rightarrow 0 \text { as } n \rightarrow \infty .
$$

We do not assume that the filtration is normal.

Proof. Define a normal filtration $\mathcal{G}_{t}:=\bigcap_{\varepsilon>0} \sigma\left(\mathcal{F}_{t+\varepsilon}, \mathcal{N}\right)$, where $\mathcal{N}$ is the collection of $P$-null sets in $\overline{\mathcal{F}}^{P}$. General theory tells us that there is a $\left(\mathcal{G}_{t}\right)$-predictable process $p(s, \omega)$ with

$$
Z(s, \omega)=p(s, \omega), \quad \mathrm{d} s \otimes P-\text { a.e. on }[0, T] \times \Omega .
$$

This result uses the optional projection theorem; see [1]. Then Proposition 2.3.8 of [4] shows that $p(s, w)$ can be approximated in $L^{2}\left([0, T] \times \Omega, \mathcal{P}_{T}, \mathrm{~d} s \times P\right)$ by elementary processes, i.e., those of the form $\sum_{m=0}^{j-1} \Phi_{m}(\omega) 1_{\left(t_{m}, t_{m+1}\right]}(s)$ where $0=t_{0}<t_{1}<\cdots<t_{j} \leqslant T$ and $\Phi_{m}$ is $\mathcal{G}_{t_{m}}$ measurable for each $j$. Here $\mathcal{P}_{T}$ is the $\sigma$-algebra generated by such elementary processes.

Suppose $r<t$ and $\Phi$ is $\mathcal{G}_{r}$ measurable, and consider the elementary $\left(\mathcal{G}_{t}\right)$-predictable process $\Phi(\omega) 1_{(r, t]}(s)$. This process can be approximated in $L^{2}$ by the sequence $\Phi(\omega) 1_{(r+1 / n, t]}(s)$ for large $n$. Since $\Phi$ is $\sigma\left(\mathcal{F}_{r+1 / n}, \mathcal{N}\right)$ measurable for each $n$, the approximants are $\sigma\left(\mathcal{F}_{t}, \mathcal{N}\right)$-predictable processes. 
But if $\Phi(\omega) 1_{(r, t]}(s)$ is $\sigma\left(\mathcal{F}_{t}, \mathcal{N}\right)$-predictable, then $\Phi$ is $\sigma\left(\mathcal{F}_{r}, \mathcal{N}\right)$ measurable, so there is a $\mathcal{F}_{r}$ measurable function $\Psi$ and a $\mathcal{F}_{r}$ measurable set $N$ so that $(\omega: \Phi(\omega) \neq \Psi(\omega)) \subseteq N$, and $P(N)=0$. Then the process $\Psi(\omega) 1_{(r, t]}(s)$ is an $\left(\mathcal{F}_{t}\right)$-predictable version of $\Phi(\omega) 1_{(r, t]}(s)$. Combining these results shows that every elementary $\left(\mathcal{G}_{t}\right)$-predictable process can be approximated in $L^{2}$ by elementary $\left(\mathcal{F}_{t}\right)$-predictable processes. This gives the result.

Suppose that $\sigma(s, w)$ is $L_{2}(U, H)$-valued, measurable, and adapted on $\left(\mathbb{B}, \mathcal{B}(\mathbb{B}),\left(\mathcal{B}_{t}(\mathbb{B})\right)\right)$, and consider the $\mathcal{B}(\mathbb{B}) \backslash \mathcal{B}([0, \infty])$ measurable map $w \mapsto \int_{0}^{t}\|\sigma(s, w)\|^{2} \mathrm{~d} s$. Since $\sigma$ is adapted, for $s \leqslant t$ we have $\sigma(s, w)=\sigma\left(s, w^{t}\right)$. Therefore

$$
\int_{0}^{t}\|\sigma(s, w)\|^{2} \mathrm{~d} s=\int_{0}^{t}\left\|\sigma\left(s, w^{t}\right)\right\|^{2} \mathrm{~d} s
$$

and Corollary 1.3 shows that this map is $\mathcal{B}_{t}(\mathbb{B}) \backslash \mathcal{B}\left(L_{2}(U, H)\right)$ measurable.

For $k \geqslant 1$ define $\tau_{k}(w)=\inf \left(t \geqslant 0: \int_{0}^{t}\|\sigma(s, w)\|^{2} \mathrm{~d} s \geqslant k\right)$. Since $t \mapsto \int_{0}^{t}\|\sigma(s, w)\|^{2} \mathrm{~d} s$ is continuous into $[0, \infty]$ for $t>0$, we see that $\left(w: \tau_{k}(w) \leqslant t\right) \in \mathcal{B}_{t}(\mathbb{B})$ for $t>0$. Also note that $\tau_{k}(w) \uparrow \tau(w):=\inf \left(t \geqslant 0: \int_{0}^{t}\|\sigma(s, w)\|^{2} \mathrm{~d} s=\infty\right)$ pointwise on $\mathbb{B}$, where the infimum of the empty set is taken to be infinity.

For any probability measure $\nu$ on $\mathcal{B}(\mathbb{B})$, and every $k \geqslant 1,1_{\left(0, \tau_{k}(w)\right]}(s) \sigma(s, w)$ is a measurable, adapted, $L_{2}(U, H)$-valued process with

$$
\int_{\mathbb{B}} \int_{0}^{T}\left\|1_{\left(0, \tau_{k}(w)\right]}(s) \sigma(s, w)\right\|^{2} \mathrm{~d} s \nu(\mathrm{d} w) \leqslant k<\infty .
$$

Therefore, from Lemma 2.5 and by taking a subsquence, we can find elementary predictable processes $p_{n}^{k}$ so that

$$
\sum_{n=1}^{\infty} \int_{\mathbb{B}} \int_{0}^{T}\left\|1_{\left(0, \tau_{k}(w)\right]}(s) \sigma(s, w)-p_{n}^{k}(s, w)\right\|^{2} \mathrm{~d} s \nu(\mathrm{d} w)<\infty .
$$

Fix a probability measure $\mu$ on $(H, \mathcal{B}(H))$ and let $(X, W)$ with stochastic basis $\left(\Omega, \mathcal{F}, P,\left(\mathcal{F}_{t}\right)\right)$ be a weak solution to (1.1) with initial distribution $\mu$. In particular we assume that $\int_{0}^{t}\|\sigma(s, X)\|^{2} \mathrm{~d} s<$ $\infty P$-a.e. for all $t \in(0, \infty)$. Recall that $P_{\mu}=P \circ(X(0), X, W)^{-1}$, and that $K_{\mu}\left((x, w), \mathrm{d} w_{1}\right)$ is the regular conditional distribution of $X$ given $X(0)=x$ and $W=w$.

We aim to give a pointwise definition for the stochastic integral $\int_{0}^{T} \sigma(s, X) \mathrm{d} W(s)$ at the fixed time $T$. Fix $k \geqslant 1$ and let $p_{n}^{k}$ be the elementary predictable processes above (for $\nu=P^{X}$ ), and define the stochastic integral as usual. That is, if $p_{n}^{k}(s, w)=\sum_{m=0}^{j-1} \Phi_{m}(w) 1_{\left(t_{m}, t_{m+1}\right]}(s)$, then

$$
I_{n}^{k}\left(w_{1}, w\right):=\int_{0}^{T} p_{n}^{k}\left(s, w_{1}\right) \mathrm{d} w(s)=\sum_{m=0}^{j-1} \Phi_{m}\left(w_{1}\right)\left[w\left(t_{m+1}\right)-w\left(t_{m}\right)\right] .
$$

Because of the isometry for stochastic integrals, and the summation condition in (2.5) we see that $\lim _{n \rightarrow \infty} I_{n}^{k}\left(w_{1}, w\right)$ exists in $L^{2}\left(P_{\mu}\right)$ and $P_{\mu}$-almost everywhere. Define

$$
I^{k}\left(w_{1}, w\right):= \begin{cases}\lim _{n} I_{n}^{k}\left(w_{1}, w\right) & \text { if the limit exists } \\ 0 & \text { otherwise }\end{cases}
$$

and then put

$$
I\left(w_{1}, w\right):=\sum_{k=1}^{\infty} 1_{\left(\tau_{k-1}\left(w_{1}\right)<T \leqslant \tau_{k}\left(w_{1}\right)\right)} I^{k}\left(w_{1}, w\right) .
$$

If we map back onto the (normal) stochastic basis $\left(\Omega, \mathcal{F}, P,\left(\mathcal{F}_{t}\right)\right)$, we find that $\tau_{k}(X)$ is a sequence of $\left(\mathcal{F}_{t}\right)$-stopping times with $\tau_{k}(X) \uparrow \infty P$-a.e. Also $p_{n}^{k}(X)$ are $\left(\mathcal{F}_{t}\right)$-predictable processes that converge in $L^{2}([0, T] \times \Omega, \mathrm{d} s \times P)$ to $1_{\left(0, \tau_{k}(X)\right]} \sigma(s, X)$, so that $I_{n}^{k}(X, W)$ converge in $L^{2}(\Omega, P)$ to the stochastic integral $\int_{0}^{T} 1_{\left(0, \tau_{k}(X)\right]} \sigma(s, X) \mathrm{d} W(s)$. Putting these pieces together shows that $I(X, W)$ is a version of the stochastic integral $\int_{0}^{T} \sigma(s, X) \mathrm{d} W(s)$. 
Lemma 2.6. There exists $N \in \mathcal{B}(H)$ with $\mu(N)=0$ so that for $x \notin N, I\left(\Pi_{1}, \Pi_{3}\right)$ is a $Q_{x}$-version of the stochastic integral $\int_{0}^{T} \sigma\left(s, \Pi_{1}\right) \mathrm{d} \Pi_{3}(s)$ on the stochastic basis $\left(\tilde{\Omega}, \tilde{\mathcal{F}}^{x}, Q_{x},\left(\tilde{\mathcal{F}}_{t}^{x}\right)\right)$.

Proof. Since $\left(\tilde{\mathcal{F}}_{t}^{x}\right)$ is a right continuous filtration, it is easy to see that $\tau_{k}\left(\Pi_{1}\right)$ is a sequence of $\left(\tilde{\mathcal{F}}_{t}^{x}\right)$-stopping times. If we define $A=\left(w \in \mathbb{B}: \lim _{k} \tau_{k}(w)=\infty\right)$, then

$$
1=P(X \in A)=\int_{H} Q_{x}\left(\Pi_{1} \in A\right) \mu(\mathrm{d} x)
$$

so there exists $N_{1} \in \mathcal{B}(H)$ with $\mu\left(N_{1}\right)=0$ so that for $x \notin N_{1}$, we have $Q_{x}\left(\Pi_{1} \in A\right)=1$.

Also if we define $p_{n}^{k}$ as in Lemma 2.5 using $\nu=P^{X}$, then $p_{n}^{k}\left(s, \Pi_{1}\right)$ are $\left(\tilde{\mathcal{F}}_{t}^{x}\right)$-predictable processes with

$$
\begin{aligned}
& \int_{H} \int_{\tilde{\Omega}} \int_{0}^{T}\left\|1_{\left(0, \tau_{k}\left(\Pi_{1}\right)\right]}(s) \sigma\left(s, \Pi_{1}\right)-p_{n}^{k}\left(s, \Pi_{1}\right)\right\|^{2} \mathrm{~d} s d Q_{x} \mu(\mathrm{d} x) \\
& \quad=\int_{H} \int_{\mathbb{W}_{0}} \int_{\mathbb{B}} \int_{0}^{T}\left\|1_{\left(0, \tau_{k}\left(w_{1}\right)\right]}(s) \sigma\left(s, w_{1}\right)-p_{n}^{k}\left(s, w_{1}\right)\right\|^{2} \mathrm{~d} s K_{\mu}\left((x, w), \mathrm{d} w_{1}\right) P^{W}(\mathrm{~d} w) \mu(\mathrm{d} x) \\
& =\int_{\mathbb{B}} \int_{0}^{T}\left\|1_{\left(0, \tau_{k}\left(w_{1}\right)\right]}(s) \sigma\left(s, w_{1}\right)-p_{n}^{k}\left(s, w_{1}\right)\right\|^{2} \mathrm{~d} s \nu\left(\mathrm{d} w_{1}\right) .
\end{aligned}
$$

From the summability in (2.5) we see there is $N_{2} \in \mathcal{B}(H)$ with $\mu\left(N_{2}\right)=0$ so that for $x \notin N_{2}$,

$$
\int_{\tilde{\Omega}} \int_{0}^{T}\left\|1_{\left(0, \tau_{k}\left(\Pi_{1}\right)\right]}(s) \sigma\left(s, \Pi_{1}\right)-p_{n}^{k}\left(s, \Pi_{1}\right)\right\|^{2} \mathrm{~d} s \mathrm{~d} Q_{x} \rightarrow 0 .
$$

As in Lemma 2.4, let $N_{3} \in \mathcal{B}(H)$ with $\mu\left(N_{3}\right)=0$ so that for $x \notin N_{3}, \Pi_{3}$ is an $\left(\tilde{\mathcal{F}}_{t}^{x}\right)$-Wiener process on $\left(\tilde{\Omega}, \tilde{\mathcal{F}}^{x}, Q_{x}\right)$. Now the summability in $(2.5)$ and the isometry for stochastic integrals means that

$$
\int_{H} \int_{\tilde{\Omega}} \sum_{n=1}^{\infty}\left\|I_{n}^{k}\left(\Pi_{1}, \Pi_{3}\right)-I^{k}\left(\Pi_{1}, \Pi_{3}\right)\right\|^{2} \mathrm{~d} Q_{x} \mu(\mathrm{d} x)<\infty,
$$

so that there is $N_{4} \in \mathcal{B}(H)$ with $\mu\left(N_{4}\right)=0$ so that for $x \notin N_{4}$, we have $I^{k}\left(\Pi_{1}, \Pi_{3}\right)=\lim _{n} I_{n}^{k}\left(\Pi_{1}, \Pi_{3}\right)$ $Q_{x}$-a.e.

Now set $N=N_{1} \cup N_{2} \cup N_{3} \cup N_{4}$ and take $x \notin N$. Then on the stochastic basis $\left(\tilde{\Omega}, \tilde{\mathcal{F}}^{x}, Q_{x}\right)$, $\Pi_{3}$ is a Wiener process, $p_{n}^{k}\left(s, \Pi_{1}\right)$ are predictable processes that converge to the adapted process $1_{\left(0, \tau_{k}\left(\Pi_{1}\right)\right]}(s) \sigma\left(s, \Pi_{1}\right)$ in $L^{2}\left([0, T] \times \tilde{\Omega}, \mathrm{d} s \times Q_{x} ; H\right)$. So, on the one hand,

$$
I_{n}^{k}\left(\Pi_{1}, \Pi_{3}\right) \rightarrow \int_{0}^{T} 1_{\left(0, \tau_{k}\left(\Pi_{1}\right)\right]}(s) \sigma\left(s, \Pi_{1}\right) \mathrm{d} \Pi_{3}(s) \text { in } L^{2}\left(\tilde{\Omega}, Q_{x} ; H\right),
$$

while, on the other hand,

$$
I_{n}^{k}\left(\Pi_{1}, \Pi_{3}\right) \rightarrow I^{k}\left(\Pi_{1}, \Pi_{3}\right) Q_{x^{-} \text {-a.e. }}
$$

We conclude that $I^{k}\left(\Pi_{1}, \Pi_{3}\right)$ is a version of the stochastic integral $\int_{0}^{T} 1_{\left(0, \tau_{k}\left(\Pi_{1}\right)\right]}(s) \sigma\left(s, \Pi_{1}\right) \mathrm{d} \Pi_{3}(s)$. Adding these up as in (2.6), we find that $I\left(\Pi_{1}, \Pi_{3}\right)$ is a version of the stochastic integral $\int_{0}^{T} \sigma\left(s, \Pi_{1}\right) \mathrm{d} \Pi_{3}(s)$.

Lemma 2.7. There exists $N^{\prime} \in \mathcal{B}(H)$ with $\mu\left(N^{\prime}\right)=0$ such that for all $x \notin N^{\prime},\left(\Pi_{1}, \Pi_{3}\right)$ and $\left(\Pi_{2}, \Pi_{3}\right)$ with stochastic basis $\left(\tilde{\Omega}, \tilde{\mathcal{F}}^{x}, Q_{x},\left(\tilde{\mathcal{F}}_{t}^{x}\right)\right)$ are weak solutions of $(1.1)$ such that

$$
\Pi_{1}(0)=\Pi_{2}(0)=x \quad Q_{x} \text {-a.e. }
$$

therefore, $\Pi_{1}=\Pi_{2} Q_{x}$-a.e. 
Proof. Let $I_{T}$ denote the function defined in (2.6). Now define a subset of $\mathbb{B} \times \mathbb{W}_{0}$ as follows

$$
\begin{aligned}
& A:=\left(\left(w_{1}, w\right): \int_{0}^{n}\left|b\left(s, w_{1}\right)\right| \mathrm{d} s<\infty \text { for } n \geqslant 1,\right. \\
& \left.\quad \text { and } w_{1}(T)=w_{1}(0)+\int_{0}^{T} b\left(s, w_{1}\right) \mathrm{d} s+I_{T}\left(w_{1}, w\right) \text { for } T \in \mathbb{Q} \cap(0, \infty)\right) .
\end{aligned}
$$

Since $(X, W)$ is a solution of the equation, we have

$$
1=P_{\mu}((X, W) \in A)=\int_{H} Q_{x}\left(\left(\Pi_{1}, \Pi_{3}\right) \in A\right) \mu(\mathrm{d} x)=\int_{H} Q_{x}\left(\left(\Pi_{2}, \Pi_{3}\right) \in A\right) \mu(\mathrm{d} x) .
$$

So there exists $N_{5} \in \mathcal{B}(H)$ with $\mu\left(N_{5}\right)=0$ so that for $x \notin N_{5}$, we have

$$
Q_{x}\left(\left(\Pi_{1}, \Pi_{3}\right) \in A\right)=Q_{x}\left(\left(\Pi_{2}, \Pi_{3}\right) \in A\right)=1 .
$$

Finally,

$$
1=P_{\mu}\left(w_{1}(0)=x\right)=\int_{H} Q_{x}\left(\Pi_{1}(0)=x\right) \mu(\mathrm{d} x)=\int_{H} Q_{x}\left(\Pi_{2}(0)=x\right) \mu(\mathrm{d} x),
$$

so there is $N_{6} \in \mathcal{B}(H)$ with $\mu\left(N_{6}\right)=0$ so that for $x \notin N_{6}$, we have $Q_{x}\left(\Pi_{1}(0)=\Pi_{2}(0)=x\right)=1$. Take $N^{\prime}:=N \cup N_{5} \cup N_{6}$, where $N$ is the null set defined in Lemma 2.6.

Hence, the first assertion follows. The second then follows by the pathwise uniqueness assumption in condition (ii) of the theorem.

Lemma 2.8. There exists a $\overline{\mathcal{B}(H) \otimes \mathcal{B}\left(\mathbb{W}_{0}\right)}{ }^{\mu \otimes P^{Q}} / \mathcal{B}(\mathbb{B})$-measurable map

$$
F_{\mu}: H \times \mathbb{W}_{0} \rightarrow \mathbb{B}
$$

such that

$$
\begin{gathered}
K_{\mu}((x, w), \cdot)=\delta_{F_{\mu}(x, w)} \\
\left(=\text { Dirac measure on } \mathcal{B}(\mathbb{B}) \text { with mass in } F_{\mu}(x, w)\right)
\end{gathered}
$$

for $\mu \otimes P^{Q}$-a.e. $(x, w) \in H \times \mathbb{W}_{0}$. Furthermore, $F_{\mu}$ is $\overline{\mathcal{B}(H) \otimes \mathcal{B}_{t}\left(\mathbb{W}_{0}\right)}{ }^{\mu \otimes P^{Q}} / \mathcal{B}_{t}(\mathbb{B})$-measurable for all $t \in[0, \infty)$, where $\overline{\mathcal{B}(H) \otimes \mathcal{B}_{t}\left(\mathbb{W}_{0}\right)}{ }^{\mu \otimes P^{Q}}$ denotes the completion with respect to $\mu \otimes P^{Q}$ in $\mathcal{B}(H) \otimes$ $\mathcal{B}\left(\mathbb{W}_{0}\right)$.

Proof. By Lemma 2.7 for all $x \in N_{1}^{c}$, we have

$$
1=Q_{x}\left(\left\{\Pi_{1}=\Pi_{2}\right\}\right)=\int_{\mathbb{W}_{0}} \int_{\mathbb{B}} \int_{\mathbb{B}} 1_{D}\left(w_{1}, w_{2}\right) K_{\mu}\left((x, w), \mathrm{d} w_{1}\right) K_{\mu}\left((x, w), \mathrm{d} w_{2}\right) P^{Q}(\mathrm{~d} w),
$$

where $D:=\left\{\left(w_{1}, w_{1}\right) \in \mathbb{B} \times \mathbb{B} \mid w_{1} \in \mathbb{B}\right\}$. Hence by Lemma 2.2 there exists $N \in \mathcal{B}(H) \otimes \mathcal{B}\left(\mathbb{W}_{0}\right)$ such that $\mu \otimes P^{Q}(N)=0$ and for all $(x, w) \in N^{c}$ there exists $F_{\mu}(x, w) \in \mathbb{B}$ such that

$$
K_{\mu}\left((x, w), \mathrm{d} w_{1}\right)=\delta_{F_{\mu}(x, w)}\left(\mathrm{d} w_{1}\right) .
$$

Set $F_{\mu}(x, w):=0$, if $(x, w) \in N$. Let $A \in \mathcal{B}(\mathbb{B})$. Then

$$
\left\{F_{\mu} \in A\right\}=\left(\left\{F_{\mu} \in A\right\} \cap N\right) \cup\left(\left\{K_{\mu}(\cdot, A)=1\right\} \cap N^{c}\right)
$$

and the measurability properties of $F_{\mu}$ follow from Lemma 2.3.

Having defined the mapping $F_{\mu}$ let us check the conditions of Definition 1.8 and Definition 1.9. 
Lemma 2.9. We have

$$
X=F_{\mu}(X(0), \bar{W}) \quad P \text {-a.e. }
$$

Proof. By Lemmas 2.3 and 2.8 we have

$$
P\left(\left\{X=F_{\mu}(X(0), \bar{W})\right\}\right)=\int_{H} \int_{\mathbb{W}_{0}} \int_{\mathbb{B}} 1_{\left\{w_{1}=F_{\mu}(x, w)\right\}}\left(x, w_{1}, w\right) \delta_{F_{\mu}(x, w)}\left(\mathrm{d} w_{1}\right) P^{Q}(\mathrm{~d} w) \mu(\mathrm{d} x)=1 .
$$

Let $W^{\prime}$ be another standard $\mathbb{R}^{\infty}$-Wiener process on a stochastic basis $\left(\Omega^{\prime}, \mathcal{F}^{\prime}, P^{\prime},\left(\mathcal{F}_{t}^{\prime}\right)\right)$ and $\xi: \Omega^{\prime} \rightarrow H$ an $\mathcal{F}_{0}^{\prime} / \mathcal{B}(H)$-measurable map and $\mu:=P^{\prime} \circ \xi^{-1}$. Let $F_{\mu}$ be as above and set

$$
X^{\prime}:=F_{\mu}\left(\xi, \bar{W}^{\prime}\right)
$$

Lemma 2.10. $\left(X^{\prime}, W^{\prime}\right)$ is a weak solution to (1.1) with $X^{\prime}(0)=\xi P^{\prime}$-a.s..

Proof. By the measurability properties of $F_{\mu}$ (cf. Lemma 2.8) it follows that $X^{\prime}$ is adapted. We have

$$
\begin{aligned}
P^{\prime}\left(\left\{\xi=X^{\prime}(0)\right\}\right) & =P^{\prime}\left(\left\{\xi=F_{\mu}\left(\xi, \bar{W}^{\prime}\right)(0)\right\}\right)=\mu \otimes P^{Q}\left(\left\{(x, w) \in H \times \mathbb{W}_{0} \mid x=F_{\mu}(x, w)(0)\right\}\right) \\
& =P\left(\left\{X(0)=F_{\mu}(X(0), \bar{W})(0)\right\}\right)=1,
\end{aligned}
$$

where we used Lemma 2.9 in the last step.

To see that $\left(X^{\prime}, W^{\prime}\right)$ is a weak solution we consider the set $A \in \mathcal{B}(H) \otimes \mathcal{B}(\mathbb{B}) \otimes \mathcal{B}\left(\mathbb{W}_{0}\right)$ defined in the proof of Lemma 2.7. We have to show that

$$
P^{\prime}\left(\left\{\left(X^{\prime}(0), X^{\prime}, \bar{W}^{\prime}\right) \in A\right\}\right)=1 .
$$

But since $X^{\prime}(0)=\xi$ is $P^{\prime}$-independent of $\bar{W}^{\prime}$, we have

$$
\begin{aligned}
\int 1_{A} & \left(X^{\prime}(0), F_{\mu}\left(X^{\prime}(0), \bar{W}^{\prime}\right), \bar{W}^{\prime}\right) \mathrm{d} P^{\prime}=\int_{H} \int_{\mathbb{W}_{0}} 1_{A}\left(x, F_{\mu}(x, w), w\right) P^{Q}(\mathrm{~d} w) \mu(\mathrm{d} x) \\
= & \int_{H} \int_{\mathbb{W}_{0}} \int_{\mathbb{B}} 1_{A}\left(x, w_{1}, w\right) \delta_{F_{\mu}(x, w)}\left(\mathrm{d} w_{1}\right) P^{Q}(\mathrm{~d} w) \mu(\mathrm{d} x)=\int 1_{A}\left(x, w_{1}, w\right) P_{\mu}\left(\mathrm{d} x, \mathrm{~d} w_{1}, \mathrm{~d} w\right) \\
= & P(\{(X(0), X, \bar{W}) \in A\})=1,
\end{aligned}
$$

where we used Lemmas 2.3 and 2.8 in the second to last step.

To complete the proof we still have to construct $F \in \hat{\mathcal{E}}$ and to check the adaptiveness conditions on it. Below we shall apply what we have obtained above now also to $\delta_{x}$ replacing $\mu$. So, for each $x \in H$ we have a function $F_{\delta_{x}}$. Now define

$$
F(x, w):=F_{\delta_{x}}(x, w), x \in H, w \in \mathbb{W}_{0} .
$$

The proof of Theorem 2.1 is then completed by the following lemma.

Lemma 2.11. Let $\mu$ be a probability measure on $(H, \mathcal{B}(H))$ and $F_{\mu}: H \times \mathbb{W}_{0} \rightarrow \mathbb{B}$ as constructed in Lemma 2.8. Then for $\mu$-a.e. $x \in H$

$$
F(x, \cdot)=F_{\mu}(x, \cdot) \quad P^{Q}-\text { a.e. }
$$

Furthermore, $F(x, \cdot)$ is $\overline{\mathcal{B}_{t}\left(\mathbb{W}_{0}\right)} P^{Q} / \mathcal{B}_{t}(\mathbb{B})$-measurable for all $x \in H, t \in[0, \infty)$, where $\overline{\mathcal{B}_{t}\left(\mathbb{W}_{0}\right)} P^{Q}$ denotes the completion of $\mathcal{B}_{t}\left(\mathbb{W}_{0}\right)$ with respect to $P^{Q}$ in $B\left(\mathbb{W}_{0}\right)$.

In particular, Conditions 1 and 2 in Definition 1.9 hold. 
Proof. Let

$$
\begin{gathered}
\bar{\Omega}:=H \times \mathbb{B} \times \mathbb{W}_{0}, \\
\overline{\mathcal{F}}:=\mathcal{B}(H) \otimes \mathcal{B}(\mathbb{B}) \otimes \mathcal{B}\left(\mathbb{W}_{0}\right)
\end{gathered}
$$

and fix $x \in H$. Define a measure $\bar{Q}_{x}$ on $(\bar{\Omega}, \overline{\mathcal{F}})$ by

$$
\bar{Q}_{x}(A):=\int_{H} \int_{\mathbb{W}_{0}} \int_{\mathbb{B}} 1_{A}\left(z, w_{1}, w\right) K_{\mu}\left((z, w), \mathrm{d} w_{1}\right) P^{Q}(\mathrm{~d} w) \delta_{x}(\mathrm{~d} z)
$$

with $K_{\mu}$ as in Lemma 2.3. Consider the stochastic basis $\left(\bar{\Omega}, \overline{\mathcal{F}}^{x}, \bar{Q}_{x},\left(\overline{\mathcal{F}}_{t}^{x}\right)\right)$ where

$$
\begin{gathered}
\overline{\mathcal{F}}^{x}:=\overline{\mathcal{B}(H) \otimes \mathcal{B}(\mathbb{B}) \otimes \mathcal{B}\left(\mathbb{W}_{0}\right)} \bar{Q} x \\
\overline{\mathcal{F}}_{t}^{x}:=\bigcap_{\varepsilon>0} \sigma\left(\mathcal{B}(H) \otimes \mathcal{B}_{t+\varepsilon}(\mathbb{B}) \otimes \mathcal{B}_{t+\varepsilon}\left(\mathbb{W}_{0}\right), \overline{\mathcal{N}}_{x}\right),
\end{gathered}
$$

where $\overline{\mathcal{N}}_{x}:=\left\{N \in \overline{\mathcal{F}}^{x} \mid \bar{Q}_{x}(N)=0\right\}$. As in the proof of Lemma 2.7 one shows that $\left(\Pi, \Pi_{3}\right)$ on $\left(\bar{\Omega}, \overline{\mathcal{F}}^{x}, \bar{Q}_{x},\left(\overline{\mathcal{F}}_{t}^{x}\right)\right)$ is a weak solution to $(1.1)$ with $\Pi(0)=x \bar{Q}_{x}$-a.e. Here

$$
\begin{aligned}
& \Pi_{0}: H \times \mathbb{B} \times \mathbb{W}_{0} \rightarrow H,\left(x, w_{1}, w\right) \mapsto x, \\
& \Pi: H \times \mathbb{B} \times \mathbb{W}_{0} \rightarrow \mathbb{B},\left(x, w_{1}, w\right) \mapsto w_{1}, \\
& \Pi_{3}: H \times \mathbb{B} \times \mathbb{W}_{0} \rightarrow \mathbb{W}_{0},\left(x, w_{1}, w\right) \mapsto w .
\end{aligned}
$$

By Lemma $2.10\left(F_{\delta_{x}}\left(x, \Pi_{3}\right), \Pi_{3}\right)$ on the stochastic basis $\left(\bar{\Omega}, \overline{\mathcal{F}}^{x}, \bar{Q}_{x},\left(\overline{\mathcal{F}}_{t}^{x}\right)\right)$ is a weak solution to (1.1) with

$$
F_{\delta_{x}}\left(x, \Pi_{3}\right)(0)=x .
$$

Hence, by our pathwise uniqueness assumption (ii), it follows that

$$
F_{\delta_{x}}\left(x, \Pi_{3}\right)=\Pi \quad \bar{Q}_{x} \text {-a.s. }
$$

Hence, for all $A \in \mathcal{B}(H) \otimes \mathcal{B}(\mathbb{B}) \otimes \mathcal{B}\left(\mathbb{W}_{0}\right)$ by Lemma 2.8 and (2.8)

$$
\int_{H} \int_{\mathbb{W}_{0}} \int_{\mathbb{B}} 1_{A}\left(x, w_{1}, w\right) \delta_{F_{\mu}(x, w)}\left(\mathrm{d} w_{1}\right) P^{Q}(\mathrm{~d} w) \mu(\mathrm{d} x)=\int_{H} \bar{Q}_{x}(A) \mu(\mathrm{d} x) .
$$

But for each $x \in H$

$$
\begin{aligned}
\bar{Q}_{x}(A) & =\int_{\bar{\Omega}} 1_{A}\left(\Pi_{0}, F_{\delta_{x}}\left(x, \Pi_{3}\right), \Pi_{3}\right) \mathrm{d} \bar{Q}_{x}=\int_{\mathbb{W}_{0}} 1_{A}\left(x, F_{\delta_{x}}(x, w), w\right) P^{Q}(\mathrm{~d} w) \\
& =\int_{\mathbb{W}_{0}} \int_{\mathbb{B}} 1_{A}\left(x, w_{1}, w\right) \delta_{F_{\delta_{x}}(x, w)}\left(\mathrm{d} w_{1}\right) P^{Q}(\mathrm{~d} w)
\end{aligned}
$$

Since $x \mapsto \bar{Q}_{x}(A)=\int_{\mathbb{W}_{0}} \int_{\mathbb{B}} 1_{A}\left(x, w_{1}, w\right) K_{\mu}\left((x, w), \mathrm{d} w_{1}\right) P^{Q}(\mathrm{~d} w)$ is $\overline{\mathcal{B}(H)}{ }^{\mu}$-measurable, so is the right hand side of (2.9). Therefore, we can integrate with respect to $\mu$ and obtain

$$
\begin{aligned}
\int_{H} \int_{\mathbb{W}_{0}} \int_{\mathbb{B}} 1_{A}\left(x, w_{1}, w\right) \delta_{F_{\mu}(x, w)}\left(\mathrm{d} w_{1}\right) P^{Q}(\mathrm{~d} w) \mu(\mathrm{d} x) \\
\quad=\int_{\mathrm{H}} \int_{\mathbb{W}_{0}} \int_{\mathbb{B}} 1_{A}\left(x, w_{1}, w\right) \delta_{F_{\delta_{x}}(x, w)}\left(\mathrm{d} w_{1}\right) P^{Q}(\mathrm{~d} w) \mu(\mathrm{d} x),
\end{aligned}
$$

which implies the assertion.

Let $x \in H, t \in[0, \infty), A \in \mathcal{B}_{t}(\mathbb{B})$, and define

$$
\bar{F}_{\delta_{x}}:=1_{\{x\} \times \mathbb{W}_{0}} F_{\delta_{x}}
$$


Then

$$
\bar{F}_{\delta_{x}}=F_{\delta_{x}} \quad \delta_{x} \otimes P^{Q}-\text { a.e. }
$$

hence

$$
\left\{\bar{F}_{\delta_{x}} \in A\right\} \in \overline{\mathcal{B}(H) \otimes \mathcal{B}_{t}\left(\mathbb{W}_{0}\right)} \delta_{x} \otimes P^{Q}
$$

But

$$
\left\{\bar{F}_{\delta_{x}} \in A\right\}=\{x\} \times\left\{F_{\delta_{x}}(x, \cdot) \in A\right\} \cup(H \backslash\{x\}) \times\{0 \in A\},
$$

so by $(2.10)$ it follows that

$$
\left\{F_{\delta_{x}}(x, \cdot) \in A\right\} \in{\overline{\mathcal{B}_{t}\left(\mathbb{W}_{0}\right)}}^{P^{Q}}
$$

Remark 2.12. For a detailed proof of the Yamada-Watanabe Theorem in infinite dimensions in the framework of mild solutions to stochastic partial differential equations we refer to the substantial work of Martin Ondreját (see [3]).

\title{
References
}

1. Chung K.L., Williams R.J., Introduction to Stochastic Integration, 2nd ed., Birkhauser, Boston, 1990.

2. Ikeda N., Watanabe S., Stochastic differential equations and diffusion processes, $\mathbf{2 4}$ of North-Holland Mathematical Library, North-Holland Publishing Co., Amsterdam, 1981.

3. Ondreját M., Uniqueness for stochastic evolution equations in Banach spaces, Dissertationes Math. Rozprawy Mat., 2004, 426, 63.

4. Prévôt C., Röckner M., A Concise Course on Stochastic Partial Differential Equations, Lect. Notes Math., 1905, Springer, 2007.

\section{Теорема Ямади-Ватанабе для нескінченно-вимірних стохастичних еволюційних рівнянь}

\author{
М.Рьокнер ${ }^{1,2}$, Б.Шмулянд ${ }^{3}$, Ш.Жанг ${ }^{4}$ \\ 1 Факультет математики та центр BiBoS, Університет Білефельда, Білефельд, Німеччина \\ 2 Факультет математики та статистики, Університет Пурду, Західний Лафаєт, США \\ 3 Факультет математики та статистичних наук, Університет Альберта, Едмонтон, Канада \\ 4 Факультет статистики, Школа математики та статистики, Університет Нового Південного \\ Велсу,Сідней, Австралія
}

Отримано 31 січня 2008 р.

Метою цього повідомлення є подати повне і детальне доведення фундаментальної теореми ЯмадиВатанабе у нескінченно-вимірних просторах, точніше, в рамках варіаційного підходу до стохастичних диференціальних рівнянь у частинних похідних.

Ключові слова: інтегрування частинами, стохастична динаміка

PACS: 02.03.Sa, 02.50.Ey 
